\title{
Le dialogue cogénératif en contexte d'accompagnement au coenseignement en enseignement secondaire
}

\author{
Auteurs \\ Nancy Granger, université de Sherbrooke, Canada, \\ nancy.granger@usherbrooke.ca
}

Philippe Tremblay, université Laval, Canada, philippe.tremblay@fse.ulaval.ca 


\section{REVUE HYBRIDE DE L'ÉDUCATION}

\section{Résumé}

Dans la pratique du coenseignement, la planification et la négociation entre les coenseignants sont deux problématiques centrales qui peuvent être surmontées par le dialogue cogénératif. Les objectifs de cet article ont pour visées: a) d'exemplifier les cinq types de dialogues cogénératifs (de gestion, de remue-méninges, critique, directif et réflexif), et b) de comprendre comment ce type de dialogue peut servir à aux chercheursaccompagnateurs pour analyser les contenus des concertations pour la recherche, et ensuite pour guider les coenseignants dans l'action. Pour ce faire, des extraits d'entretiens ou des notes d'observation colligées au cours d'une recherche-action sur le coenseignement sont utilisés. Les résultats montrent que les dialogues sont présents lors de consultations formelles et informelles et que les rôles des chercheurs-accompagnateurs se modulent selon différentes dimensions, et ce, en vue de stimuler le développement professionnel chez les coenseignants.

Mots-clés: coenseignement, dialogue cogénératif, planification, collaboration 


\section{$\&$}

\section{REVUE HYBRIDE DE L'ÉDUCATION}

\section{Introduction}

En contexte de coenseignement, la planification de l'enseignement et la négociation des rôles entre les enseignants en contexte de classe sont deux problématiques centrales. En effet, il y a un fort consensus pour considérer que le coenseignement ne concerne pas que l'enseignement à proprement dit. La grande majorité des définitions du coenseignement s'entendent sur trois temps du coenseignement: la coplanification, le coenseignement et la coévaluation. "Le coenseignement exige des enseignants [...] qu'ils coplanifient, coinstruisent et coévaluent », et ce, sur une base régulière (Murawski, 2009, p. 10).

Il y a également consensus sur le fait que les coenseignants ont besoin de temps de planification, encore plus avant de commencer à coenseigner (Friend et Cook, 2007 ; Murawski, 2009). La planification et le temps de concertation apparaissent comme des préoccupations principales des coenseignants, surtout chez les plus novices. Par ailleurs, les enseignants expérimentés ne planifient plus vraiment en enseignement solitaire; ils utilisent le plus souvent l'habitude, un manuel ou des planifications passées. Enfin, la coplanification représente un véritable défi quand un enseignant-ressource, par exemple, est affecté à plusieurs classes et doit donc coplanifier avec plusieurs collègues. Par ailleurs, dans de nombreux guides sur le coenseignement (Hang et Rabren, 2009; Murawski, 2009; Ploessl et al., 2010 ; Sileo, 2011; Trent, Driver, Wood, Parrott, Martin, Smith et Smith, 2003), on rappelle que la première étape de la mise en œuvre réussie de la coplanification consiste à négocier les objectifs, les attentes et les rôles, ainsi que la compréhension des exigences de l'établissement scolaire (c.-à-d. les attentes de la classe et les besoins des élèves).

Engeström (1994) a constaté que la planification et la négociation qu'elle implique constituent essentiellement un processus récursif. Les situations d'enseignement-apprentissage sont développées ensemble, de manière ouverte et en spirale. En général, les coplanificateurs revisitent leur enseignement à plusieurs reprises quand ils planifient, enseignent, réfléchissent, modifient et évaluent leurs efforts pédagogiques. Les coplanificateurs n'utilisent pas nécessairement de manière classique la parole, les réunions ou des procédures décisionnelles lourdes dans le cadre de cette coplanification. Une fois que les coplanificateurs se sont familiarisés avec le style et les compétences de leurs collègues, ils peuvent construire plus facilement sur les idées des uns et des autres et faire des retours vers des points antérieurs dans la discussion, quand ils font et imaginent ensemble des situations d'enseignement/apprentissage (Engeström, 1994). En raison de la complexité du processus de planification, il est facile de comprendre pourquoi une planification réussie exige du temps, des aptitudes à la communication efficace et une confiance dans les compétences de l'autre et dans l'engagement à des objectifs communs. 


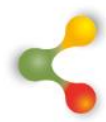

\section{REVUE HYBRIDE DE L'ÉDUCATION}

Dans le cadre de cet article, le processus de planification et de négociation de neuf binômes de coenseignement, impliquant les chercheurs-accompagnateurs, sera analysé en utilisant le concept de dialogue cogénératif (Scantlebury, Gallo-Fox et Wassel, 2008). Bien que certaines recherches commencent à s'intéresser à ce dispositif d'accompagnement au secondaire, encore peu de recherches portent sur les contenus du dialogue entre coenseignants, sur les pratiques pédagogiques et sur le développement professionnel que cette collaboration génère. Pour ce faire, des entretiens ont été réalisés avec ces enseignants, soit avant une situation d'enseignement-apprentissage, soit après des séances d'observation. L'objectif était de savoir comment ce dialogue s'opérationnalise chez les coenseignants, afin de comprendre comment il peut servir aux chercheurs-accompagnateurs, pour analyser les contenus de ces concertations pour la recherche et pour mieux guider les coenseignants dans l'action.

\section{Contexte}

Le coenseignement en enseignement secondaire est une réalité relativement nouvelle au Québec. Essentiellement, sa mise en œuvre est reliée à un accroissement des mesures inclusives/intégratives au secteur régulier, qui a conduit, entre autres, à introduire la fonction d'enseignantressource depuis 2006.

\subsection{L'intégration scolaire et les visées inclusives}

$\mathrm{Au}$ tournant du dernier millénaire, une nouvelle politique de l'adaptation scolaire a été adoptée. Cette politique a pour objectif de favoriser la scolarisation en classe ordinaire des élèves ayant des besoins spécifiques. Elle se présente comme l'une des six voies d'action définies afin de

«mettre l'organisation au service des élèves en la fondant sur l'évaluation individuelle de leurs besoins, en s'assurant qu'elle se fasse dans le milieu le plus naturel pour eux, le plus près possible de leur lieu de résidence, et en privilégiant l'intégration à la classe ordinaire " (ministère de l'Éducation du Québec, 1999, p. 23).

Aujourd'hui, plus de $20 \%$ des élèves québécois des écoles publiques sont considérés comme des élèves handicapés ou en difficulté d'adaptation ou d'apprentissage (EHDAA), alors qu'ils représentaient $10 \%$ lors de la mise en œuvre de cette politique en 2000 (MEESR, 2015). Près de $70 \%$ sont intégrés en classes ordinaires au primaire, contre $50 \%$ au secondaire (Bélanger, 2010 ; MELS, 2014). 


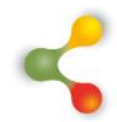

\section{REVUE HYBRIDE DE L'ÉDUCATION}

Selon un récent rapport statistique du ministère de l'Éducation et de l'Enseignement supérieur, les taux de réussite des élèves du secondaire apparaissent préoccupants (MEESR, 2015). Pour l'ensemble du Québec, le taux de diplomation et de qualification de la cohorte entrée au secondaire en 2007-2008 était de 77,7 \%, après sept ans, portant le nombre de jeunes non diplômés à $23,3 \%$, soit près du quart des élèves. En ce qui concerne les élèves en difficulté d'adaptation ou d'apprentissage (ÉDAA) qui étaient en $1^{\text {re }}$ secondaire au moment de l'adoption de la Politique de l'adaptation scolaire (1999-2000), 19 \% ont obtenu leur diplôme d'études secondaires après cinq ans, et $22 \%$ après six ans (Kalubi, 2015; Tremblay et Belley, 2017).

Cette politique souhaitant scolariser les élèves dans le milieu le plus naturel pour eux a conduit à offrir des services de soutien au secondaire (MEQ, 1999). Les orthopédagogues étant peu présents à ce degré de l'enseignement (ou de la scolarité) et les enseignants spécialisés étant affectés à d'autres services et dispositifs (ex. : classe spéciale), la mesure "enseignant-ressource» a constitué l'une des réponses au souhait de scolariser le plus grand nombre d'élèves en classe ordinaire. Ainsi, au secondaire, ce sont majoritairement les enseignants de la classe ordinaire à qui l'on confie le soutien des élèves à risque et l'intégration des élèves en difficulté d'adaptation ou d'apprentissage (EDAA) (Tremblay et Granger, 2018). Chaque année, la direction d'établissement en collaboration avec son équipe-école choisit des enseignants de classes ordinaires, qui interviendront auprès des élèves en difficulté et de leurs enseignants. Ce mandat est renouvelé annuellement selon les besoins identifiés par l'équipe-école. Pour respecter la convention collective qui balise légalement la fonction d'enseignant-ressource, ces enseignants sont "[...] libérés pour un maximum de $50 \%$ de leur tâche éducative afin d'exercer leurs fonctions » (Fédération des syndicats de l'enseignement (FSE), 2015, p. 255).

\subsection{Le coenseignement}

Dans ce contexte, enseigner à deux (un enseignant titulaire et un enseignant-ressource) apparait comme une piste de solution pour prendre en compte la diversité d'une classe ordinaire (Tremblay et Granger, soumis). Parmi les avantages répertoriés, le coenseignement permet, entre autres, une réduction du ratio enseignant/élèves dans le but d'interagir plus souvent avec les élèves en difficulté et d'offrir un enseignement plus individualisé et intensif (Friend et Cook, 2007), tout en étant moins stigmatisant (Murawski et Hughes, 2009). Toutefois, il demeure que son introduction dans les classes représente un défi important pour des coenseignants débutants ou novices. En effet, il appert que le coenseignement est une pratique complexe à mettre en place et qu'elle nécessite un temps d'appropriation entre les enseignants qui débutent dans ce modèle de service. De plus, les chercheurs s'entendent pour affirmer que le coenseignement s'applique à des degrés divers. Plusieurs 


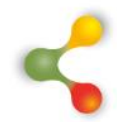

\section{REVUE HYBRIDE DE L'ÉDUCATION}

recherches soulignent que c'est la configuration «l'un enseigne/l'autre observe ou aide » qui est le plus souvent utilisée par les coenseignants (Scruggs, Mastropieri et McDuffie, 2007), et tendent à démontrer que cette configuration correspondrait à une faible réalisation de son potentiel pédagogique.

Or, pour aller plus loin et soutenir davantage d'élèves, les coenseignants doivent coplanifier, coconstruire et coévaluer, et ce, sur une base régulière (Hartnett, Weed, McCoy, Theiss et Nickens, 2013). Ces étapes supposent notamment de développer des interactions de qualité entre les deux enseignants (Friend et Cook, 2013). Trent et ses collaborateurs (2003) ont signalé que des relations de coenseignement mutuellement satisfaisantes se dessinent lorsque les enseignants se concentrent sur les aspects techniques du coenseignement, autrement dit sur l'établissement des rôles et des responsabilités, avant de s'engager dans une relation de coenseignement. Ce type de communication nécessite du temps, une connaissance des modèles de coenseignement (y compris la meilleure utilisation de chaque modèle) et des compétences en matière de négociation, non seulement pour plaider en faveur d'un enseignement adapté aux besoins des élèves, mais aussi pour mettre à profit les opportunités qui exploitent au mieux les compétences des deux enseignants. Des problèmes surviennent lorsque la phase de négociation initiale échoue. Pour y arriver, l'enseignant et l'enseignant-ressource doivent échanger sur le rôle de chacun auprès des élèves et sur leurs attentes réciproques (Granger et Tremblay, accepté). Récemment, Granger et Tremblay (2019) ont montré que, parmi les tâches des enseignants-ressources, les pratiques collaboratives impliquant des échanges et des discussions avec leurs collègues leur apportent beaucoup de satisfaction. Les enseignants-ressources qui coenseignent se sentent plus efficaces à deux. Ils mentionnent que le coenseignement leur permet davantage d'innover, de mettre en place de nouvelles stratégies et de s'ouvrir à des méthodes d'enseignement diversifiées.

La coplanification aide d'abord les coenseignants à définir clairement les buts de la collaboration. Dans cet esprit, il convient de noter que de nombreux enseignants n'ont pas été formés à des modèles collaboratifs (Kampwirth, 1999), ce qui pose des problèmes potentiellement graves au début de la mise en œuvre du coenseignement. Les coenseignants doivent comprendre comment leurs connaissances et leurs compétences facilitent l'apprentissage en coenseignement. Murata (2002) indique que les coenseignants perçoivent les habiletés et les compétences de l'autre comme contributoires à une meilleure planification et à leur développement professionnel. Puisque les enseignants-ressources sont appelés à coenseigner dans plusieurs classes et avec plusieurs de leurs collègues, la capacité à collaborer devient incontournable (Bergeron et Granger, 2016 ; Friend et Cook, 2007 ; Granger et Dubé, 2015). En ce sens, la capacité des coenseignants à communiquer et à identifier leurs compétences respectives serait favorable à un partage équitable des 


\section{$\&$}

\section{REVUE HYBRIDE DE L'ÉDUCATION}

tâches. Sans cet échange, il est plus probable qu'ils jouent le rôle d'assistants de classe (Scruggs et al., 2007) plutôt que de devenir des partenaires pédagogiques. L'expertise de chacun permet des planifications de situations d'enseignement/apprentissage plus différenciées pour répondre aux besoins de tous les élèves, en particulier de ceux ayant des besoins spécifiques (Fennick et Liddy, 2001 ; Murawski et Hugues, 2009).

Concernant la planification, Tremblay (2010) a identifié trois types de concertation dans le cadre du coenseignement, chacun dans un espace/temps différent: les concertations formelles, les concertations informelles et les concertations directes. Les premières sont des moments plus ou moins réguliers (ex. : lundi après la classe, pendant les heures sans élèves, etc.) pendant lesquels les enseignants conviennent, d'avance, de se rencontrer pour parler de certains points précis (ex. : élaboration du programme de la semaine, mise au point d'activités, etc.). Le deuxième type de concertation, les concertations informelles, sont liées à la mise en adéquation des horaires des enseignants. Différents moments de la journée scolaire des enseignants sont constitués d'heures sans classe: repas de midi, début de matinée, fin de journée, récréation, surveillance, etc. Ces moments permettent des rencontres improvisées, mais fréquentes, plus ou moins imposées par la vie en commun à l'école. Bien entendu, dans le cas d'un coenseignement intensif, les coenseignants ayant des horaires communs passent un temps important ensemble, en dehors du temps d'enseignement. Ces rencontres informelles permettent des concertations courtes au cours desquelles des rétroactions sont données, des ajustements aux activités qui ont été réalisées sont faits, des idées sont proposées pour de nouvelles activités, etc. Enfin, les concertations directes, ou in situ, concernent les discussions entre enseignants, en classe, pendant qu'ils ont la charge des élèves. II s'agit, le plus souvent, de moments où les deux enseignants se rejoignent pour discuter, pendant quelques secondes ou minutes, d'un sujet concernant les activités réalisées dans la journée ou « en direct » (rétroaction, ajustement, improvisation, etc.). II s'agit, d'une part, d'une régulation de l'activité et d'autre part, d'échanges d'information sur les élèves et l'activité en cours.

\section{Cadre conceptuel ; le dialogue cogénératif}

Bien que l'on puisse définir certains types de concertations entre coenseignants, des lieux et des temps de rencontres diversifiés pour coplanifier, il appert que les contenus de ces rencontres et leur structuration demandent d'utiliser d'autres outils pour l'analyse des échanges entre coenseignants et entre coenseignants et chercheurs-accompagnateurs. Différents auteurs ont utilisé, pour ce faire, le dialogue cogénératif. Celui-ci est défini comme une discussion formelle entre enseignants, basée sur des expériences partagées et centrées sur l'amélioration de l'enseignement et de l'apprentissage (LaVan et Beers, 2005; Scantlebury, Gallo-Fox et Wassel, 2008). Ce type de dialogue serait profitable au développement professionnel des enseignants sur les plans pédagogiques et didactiques. 


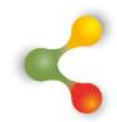

\section{REVUE HYBRIDE DE L'ÉDUCATION}

En s'écoutant les uns les autres, les enseignants ont accès à des perspectives et à des interprétations différentes de l'expérience d'enseignement et d'apprentissage (Tobin et Roth, 2006). Cet accès aux représentations mutuelles aiderait les coenseignants à mieux planifier les activités d'apprentissage en concertation (Emdin, 2007). En effet, sans coplanification, les enseignants n'auraient pas une compréhension commune de l'enseignement en classe et établiraient des objectifs divergents pour les élèves.

Scantlebury, Gallo-Fox et Wassel (2008) ont réalisé une recherche sur les dialogues cogénératifs entre les enseignants associés et leurs stagiaires, au cours de stages en enseignement. Les résultats montrent que les dialogues ont permis aux stagiaires de développer les dimensions culturelles et sociales de l'enseignement. Les stagiaires ont eu de nombreuses occasions de réfléchir à leur pratique en tant qu'enseignants débutants et ont appris à collaborer avec d'autres personnes dans les processus de planification, d'apprentissage et d'évaluation. Dans le modèle de coenseignement, les stagiaires ont interagi avec plusieurs enseignants appartenant ou non à leur discipline. À l'issue de cette expérience, les enseignants déclarent avoir aussi appris des stagiaires, notamment parce que ces derniers détenaient des connaissances disciplinaires récentes et qu'ils avaient des habiletés affirmées quant à l'utilisation des technologies en enseignement (Gallo-Fox et Scantlebury, 2006). En outre, l'accès et la mise en commun des ressources de chacun ont renforcé la conviction des stagiaires que le partage était un comportement approprié et bénéfique au sein de l'école. En revanche, les horaires chargés et le temps passé en rencontres avec les divers coenseignants se sont avérés complexes à gérer. Pour solutionner ce désagrément, du temps de coplanification a été ajouté pour que les coenseignants puissent accéder à des temps d'échange de qualité.

Pour leur part, Stith et Roth (2010) proposent une synthèse de leurs travaux sur le coenseignement et le dialogue cogénératif, en vue de soutenir leur argumentaire en faveur duquel les enseignants doivent être mieux préparés pour comprendre leur rôle complexe en classe ainsi que leur responsabilité éthique envers les élèves. Ces auteurs réfèrent plus spécifiquement à la théorie de l'activité historico-culturelle (Engeström. 1987; Leont'ev, 1978), qu'ils utilisent pour analyser et théoriser les cadres pédagogiques. Selon cette théorie, toute action est comprise comme une relation transitive entre le sujet et son objet d'activité, laquelle est médiée par les outils disponibles, par la division du travail, par la communauté et par les règles inhérentes à l'activité (Engeström, 2001). Dans le cas concret de la scolarisation, l'enseignant et ses élèves constituent les sujets. Leurs actions sur l'objet (le coenseignement) sont médiées, par exemple, par la division du travail qui leur assigne leurs rôles. Stith et Roth. (2010) mettent l'accent sur le rôle de médiateur de l'enseignant et son rôle éthique central dans la gestion des tensions qui se posent, en situation, sur les objets d'apprentissages, les approches pédagogiques préconisées, l'organisation 


\section{$\&$}

\section{REVUE HYBRIDE DE L'ÉDUCATION}

scolaire déterminée, les buts et les visées parfois contradictoires au sein de l'école ou de la classe, etc. En ce sens, le dialogue cogénératif constitue un espace privilégié pour résoudre les problèmes, favoriser le respect mutuel entre enseignants, mais aussi avec les élèves qui sont considérés également comme des acteurs qui doivent avoir le droit et l'opportunité de s'exprimer. Enfin, les auteurs soutiennent que c'est à travers ce type d'échange que les enseignants développent leur leadeurship pédagogique et parviennent à rendre les élèves plus actifs et à s'engager.

Enfin, Beltramo (2017) a mené une étude de type ethnographique sur les expériences de deux enseignants de science qui, en plus de discuter ensemble, ont créé un dialogue avec leurs élèves. Durant 16 semaines, Beltramo a animé des séances de dialogue cogénératif inspirées des études de Siry et Lang (2010) et suivies d'un temps de régulation (debriefing). II a aussi tenu un journal de bord et utilisé des captations vidéo montrant des échanges entre les enseignants et les élèves en salle de classe. Cette étude montre que le dialogue cogénératif révèle de nombreuses informations sur les étudiants, qui peuvent être utiles à l'enseignant pour adapter sa pratique. Ces informations peuvent être divisées en trois catégories soit a) les besoins en apprentissage des étudiants, b) leurs besoins sociaux, et c) leur questionnement et leurs intérêts personnels. En ce qui concerne l'apprentissage, les coenseignants s'aperçoivent qu'ils doivent clarifier le vocabulaire qu'ils utilisent et s'arrimer entre eux lorsqu'ils donnent des explications. De plus, leur dialogue révèle qu'ils doivent se questionner sur ce que l'élève est prêt à apprendre et le soutien qu'ils devront lui offrir pour aller plus loin. Les échanges ont conduit à davantage tenir compte de la zone proximale de développement de leurs élèves. De plus, favoriser des situations d'apprentissage en dyade et en groupe a contribué à combler les besoins sociaux de leurs élèves lorsqu'ils étaient en appropriation de contenu. L'apprentissage en dyade s'est d'ailleurs avéré un excellent moyen pour stimuler le questionnement réciproque préalable à des discussions en groupe. Cette façon de faire correspondait aux préférences de leurs élèves qui trouvaient plus sécurisant d'échanger avec un pair au départ. Ces résultats corroborent ceux d'autres auteurs qui soulignent l'importance de tenir compte des intérêts des élèves en créant des situations d'enseignement-apprentissage ancrées dans le réel et basées sur les questionnements générés par les élèves (Beers, 2005 ; Smagorinsky, 2013).

Pour conduire à une meilleure compréhension des besoins des élèves (Stith et Roth, 2006, 2008), le dialogue cogénératif doit s'inscrire dans une relation dialectique impliquant trois aspects. Le premier aspect est l'activité de coenseignement comme défini plus haut. Le deuxième aspect est le corespect illustrant le respect mutuel entre coenseignants qui favorise la communication et crée un environnement ouvert aux critiques constructives, au partage et à la génération de nouvelles idées, voire à une expérience fructueuse de formation continue. Le troisième aspect concerne la coresponsabilité qui suggère que chacun assume une part égale de 


\section{$\&$}

\section{REVUE HYBRIDE DE L'ÉDUCATION}

responsabilité sur tous les aspects de la classe, soit la préparation de la classe, l'enseignement, le partage de l'autorité, les élèves, les résultats et tous les autres aspects de la gestion de l'enseignement. Ces trois aspects sont interdépendants les uns des autres (Scantlebury et al., 2008). Cinq types différents de dialogues cogénératifs ont été identifiés par Scantlebury et al. (2008). Chacun de ces types de dialogue cogénératif possède des caractéristiques différentes, qui sont susceptibles de se retrouver de manière combinée au sein d'une même période d'enseignement.

1. Le dialogue cogénératif de gestion est principalement axé sur la résolution de problèmes liés à la répartition des tâches d'enseignement: la logistique des cours, l'attribution des rôles, la préparation du matériel, l'accès des élèves à des ressources complémentaires telles que la bibliothèque ou les laboratoires informatiques.

2. Le dialogue cogénératif de remue-méninges est associé au moment durant lequel les coenseignants planifient le programme, discutent de la manière de le présenter aux élèves en utilisant diverses approches pédagogiques et examinent comment ces approches devraient varier en fonction des ressources disponibles. Dans le cadre du remueméninge, les coenseignants peuvent aussi cogénérer des idées pour des pratiques d'enseignement et d'évaluation efficaces.

3. Le dialogue cogénératif critique discute des limites perçues quant au contenu ou au regard des pratiques d'enseignement mises en œuvre. Les coenseignants peuvent discuter de l'activité qui leur semble la plus appropriée pour répondre aux objectifs visés et exploiter le contenu au programme. Ce dialogue peut aider à recentrer leur travail et leurs rôles auprès des élèves.

4. Le dialogue cogénératif directif a pour but de permettre aux coenseignants de s'exprimer sur une prise d'autorité non souhaitée par l'un ou l'autre des enseignants. II permet de verbaliser comment l'un et l'autre vivent ce changement d'équité dans les rôles et de recadrer la relation.

5. Le dialogue cogénératif réflexif est représenté lorsque les coenseignants utilisent les données recueillies lors des enseignements ou des travaux d'élèves pour prendre des décisions concernant les orientations futures. II donne lieu à un dialogue coopératif réflexif au cours duquel les coenseignants discutent d'approches efficaces permettant de s'appuyer sur les connaissances des élèves pour favoriser leur réussite. II peut s'agir de se centrer davantage sur la planification des rôles de chacun, du contenu, des formules pédagogiques, des types de ressources à offrir (ex. : informatique, humaine, matérielle), etc. 


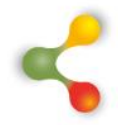

\section{REVUE HYBRIDE DE L'ÉDUCATION}

Chacun de ces types de dialogue cogénératif possède des caractéristiques différentes, qui sont susceptibles de se retrouver de manière combinée au sein d'une même période d'enseignement.

\section{Méthodologie}

Tel que décrit plus haut, le coenseignement nécessite de la concertation entre les deux enseignants. Ces espaces de dialogue peuvent contribuer au développement professionnel et à l'intercompréhension des enseignants. Dans cette recherche-action, des temps d'échanges entre enseignants et chercheurs-accompagnateurs, autour de situations observées ou à observer, ont été enregistrés ou observés, et serviront de base pour rendre visibles les interactions. La recherche-action implique que, dans le processus de construction de la recherche, tant les chercheurs-accompagnateurs que les acteurs participent à l'expérimentation. " II s'agit de recherches dans lesquelles il y a une action délibérée de transformation de la réalité et produire des connaissances concernant ces transformations » (Hugon et Seibel, 1988, p.13). De nature cyclique, le processus de recherche-action s'articule généralement en quatre grandes étapes : la planification, l'action, l'observation et la réflexion (Prud'homme, Dolbec et Guay, 2011). L'action des chercheursaccompagnateurs peut s'exercer de manière plus active à toutes les étapes du projet ou à certaines d'entre elles. Enfin, la recherche-action pour démontrer sa pertinence doit être en mesure d'évaluer et de comparer les effets de son action par rapport à une situation antérieure ou comparable. Travaillant dans le cadre d'une recherche-action, c'est-à-dire inscrite dans le réel, il faut veiller à ce que les actions entreprises ne défavorisent pas les personnes concernées.

L'objectif général de cet article consiste à illustrer comment le dialogue cogénératif peut être utilisé pour analyser l'accompagnement des chercheurs-accompagnateurs auprès de binômes de coenseignement. Plus précisément, il permet d'exemplifier et d'analyser les cinq types de dialogues cogénératifs relevés par Scantlebury, Gallo-Fox et Wassel (2008). Chacun pouvant servir de guide pour l'analyse des contenus et de l'accompagnement des coenseignants.

\subsection{Participants}

Les dialogues et les résumés présentés proviennent d'entretiens d'accompagnement réalisés avec des coenseignants $(n=18)$ issus de trois écoles secondaires du Québec participant à une recherche-action sur le déploiement du coenseignement au $1^{\mathrm{er}}$ cycle du secondaire en français et mathématiques. Les enseignants avaient entre 5 et 23 ans d'expérience en enseignement. Tous débutaient en coenseignement et souhaitaient un accompagnement. Par ailleurs, ils avaient tous au moins une période de concertation formelle après chaque cycle de neuf jours. 


\section{$\&$}

\section{REVUE HYBRIDE DE L'ÉDUCATION}

\subsection{Dispositif et outils}

Au départ, deux formations sur le coenseignement (configuration et planification) ont été offertes aux enseignants de chaque école par les deux chercheurs-accompagnateurs. Par la suite, des observations en salle de classe ont eu lieu à trois ou quatre reprises durant l'année selon un calendrier établi entre les chercheurs-accompagnateurs et les coenseignants. Chacune était suivie ou précédée d'un entretien d'accompagnement. Pour les entretiens suivant une observation, la démarche réflexive en partenariat était appliquée de manière à créer des discussions entre les enseignants dans une atmosphère de cordialité et à les amener à traduire le plus fidèlement possible les idées dominantes qui délimitaient l'expérience vécue (Boudreault et Kalubi, 2006). Dans le cas présent, il s'agissait d'avoir accès à ce que les coenseignants pensaient ou vivaient lorsqu'ils étaient deux au sein de la classe. Pour ce faire, une question ouverte était lancée en guise d'amorce au dialogue. Les coenseignants étaient alors libres de s'exprimer, de s'interpeller et de se relancer à propos les éléments abordés par l'un ou l'autre. L'un ou l'autre des chercheurs-accompagnateurs pouvait relancer par une question ou une remarque pour aider les coenseignants à cerner le sujet. L'objectif visé était qu'ils coconstruisent du sens autour de leur prestation d'enseignement et qu'ils posent ultimement un regard réflexif sur leur pratique. Pour les entretiens qui précédaient les observations, il s'agissait plutôt de leur faire expliciter, dans un premier temps, les contenus et objectifs de la situation qui sera observée, puis de participer avec eux à la coconstruction de cette activité en les questionnant et en proposant des exemples tirés de situations vécues ailleurs.

Dans cet article, des extraits enregistrés de dialogues réalisés au cours d'une recherche sur le coenseignement ainsi que des notes d'observation ont été utilisés pour illustrer et analyser les différents types de dialogues mis en œuvrent dans les accompagnements réalisés (Granger et Tremblay, 2019). Ces notes d'observation, issues des carnets de recherche, permettent de comprendre l'ensemble d'une séance ou d'une démarche. Elles ont été soumises aux enseignants pour validation et accord.

\section{Résultats}

Cette section illustre les différents types de dialogues cogénératifs (de gestion, de remue-méninge, critique, directif, réflexif) relevés lors des séances d'observation en classe ou de régulation a posteriori. Les résultats permettent, d'une part d'illustrer chaque type de dialogue, et d'autre part, de situer le rôle des chercheurs-accompagnateurs dans chacun des types de coenseignement. 


\section{$\&$}

\section{REVUE HYBRIDE DE L'ÉDUCATION}

\subsection{Dialogue cogénératif de gestion}

Ce type de dialogue est basé sur la résolution de problèmes liés à la répartition des tâches et des ressources, mais aussi sur la préparation du matériel et l'accès des élèves à des ressources complémentaires telles que la bibliothèque ou les laboratoires informatiques. Ce type de dialogue est observable lors des concertations formelles entre enseignants. II constitue un sujet important de discussion en début d'année scolaire ou de semestre (horaires, partage des rôles, etc.). Ce dialogue sert à la macroplanification des activités de l'année ou des prochaines semaines, mais également de l'espace, du matériel et du temps. En contexte inclusif, il concerne également la gestion des plans d'intervention, des adaptations et modifications qui y sont inscrites. La note d'observation 1 illustre ce type de préoccupation :

Lors des premières rencontres entre coenseignantes qui commençaient un projet de coenseignement intensif, ces dernières ont remarqué le nombre important d'élèves avec des adaptations inscrites au plan d'intervention qui leur donnaient droit à l'utilisation d'un ordinateur et de certaines fonctions d'aide lors de tâches en lecture et/ou écriture. Comme l'enseignante-ressource a également un rôle d'enseignante en adaptation scolaire, il a été décidé de lui confier les tâches liées aux adaptations technologiques. Par ailleurs, la configuration de la classe a été modifiée pour permettre une utilisation facile des ordinateurs.

Dans le cadre du dialogue cogénératif de gestion, le rôle des chercheurs-accompagnateurs est celui de conseiller en début d'année, puis d'observateur lors de visites en classe et lors des rétroactions après une séance de coenseignement. En début d'année, il peut attirer l'attention sur différents aspects à ne pas oublier dans cette gestion (horaires, plan d'intervention, évaluations, etc.). Lors des observations en classe, les notes prises servent à formuler des relances aux questionnements des enseignants ou lors de l'accompagnement du dialogue cogénératif directif quand des problèmes surviennent. Cette observation est également utile lors du dialogue cogénératif critique et réflexif au cours duquel les rôles sont redéfinis à la suite de l'expérience. L'extrait 1 ci-dessous illustre ce questionnement des chercheurs-accompagnateurs dans la répartition des rôles en fonction des forces de chacun, au sein de la classe.

Accompagnateur : Quelle place fais-tu à l'enseignant-ressource dans ta classe?

Enseignante titulaire : On compartimentait les tâches. J'ai laissé à C. le soin de l'aspect technique. Je t'ai laissé faire là-dedans et aussi la gestion 


\section{8}

\section{REVUE HYBRIDE DE L'ÉDUCATION}

du temps. Je trouvais que t'étais bonne là-dedans. Elle mettait le temps au tableau interactif, ça aidait les élèves.

Enseignante ressource : On compartimentait peut-être plus quand j'étais là, au niveau du temps.

Enseignante titulaire : Au niveau du temps et aussi la consultation des élèves. Moi, l'aide que j'apportais, c'était peut-être un peu plus en profondeur. [...] parce que c'est moi qui corrige au bout de la ligne. Et je connais ma matière; je la connais du bout des doigts. Donc, quand j'avais besoin de rencontrer des élèves en regardant leur texte en profondeur, bien ça demandait plus de temps, plus de concentration; donc pendant ce temps-là, C. supervisait la classe en entier. Donc, ça c'était vraiment aidant.

On peut également observer ce type de dialogue, in situ, lors de l'enseignement proprement dit, où des microconcertations et des signes non verbaux sont utilisés pour gérer et ajuster l'activité en cours. Par exemple, deux enseignants se parlent quelques secondes pour prévoir la transition entre deux activités ou encore pour mettre en place une table de remédiation animée par l'un des coenseignants pour quelques élèves en plus grand besoin, tandis que l'autre prend en charge le reste du groupe. Cela concerne aussi la gestion du matériel pédagogique (cahiers, ordinateurs portables), la gestion de l'espace (regroupement stratégique d'un coin de concertation ou pour isoler des élèves) ou encore, celle du temps (adapter la panification prévue à l'activité réelle ou selon des attentes différenciées).

\subsection{Dialogue de remue-méninge}

Le remue-méninge permet aux coenseignants de cogénérer des idées pour mettre en œuvre des pratiques d'enseignement et d'évaluation nouvelles et/ou efficaces. On observe ce type de dialogue essentiellement lors des concertations formelles, bien que la discussion qui suit une activité (voir dialogue cogénératif critique) serve souvent de base à cette concertation. II s'agit essentiellement de « sortir des ornières » de certaines habitudes, de vaincre une difficulté nouvelle et chronique, de trouver une solution nouvelle, etc. Dans l'extrait 2, deux coenseignants de français souhaitaient travailler la structure du texte narratif avec les élèves qui éprouvent de la difficulté à restituer l'histoire selon la séquence attendue, soit: la situation initiale, l'élément déclencheur, les péripéties, le dénouement et la situation finale. Ce dialogue, survenu à la suite d'un cours qui a moins bien fonctionné ou d'une insatisfaction de l'enseignante titulaire, permettait aux deux enseignants de créer une ouverture dans un fonctionnement plus conventionnel, voire normatif.

Enseignant titulaire : À partir du besoin des élèves, qu'est-ce qu'on pourrait mettre en place? 


\section{$\&$}

\section{REVUE HYBRIDE DE L'ÉDUCATION}

Enseignant-ressource : On part du fait qu'on a des textes à lire et on se questionne pour savoir comment on va faire pour ne pas qu'ils perdent le sens.

Enseignant titulaire : Il y aurait peut-être moyen de travailler en îlot avec les différents textes.

Je travaille beaucoup avec l'organisateur graphique en couleur pour faire le schéma narratif. On pourrait le faire en station avec les différents textes... On pourrait reprendre le même organisateur sous forme de casse-tête ou une construction visuelle, de couleur...

Enseignant-ressource : Moi je pensais à quelque chose de plus de base que ça... On avait déjà découpé des sections de textes qu'ils devaient remettre en ordre.

Les entretiens précédant une observation, avec la participation active ou non des chercheurs-accompagnateurs dans le but de les accompagner dans de nouvelles pratiques, constituent ici la principale source de ce type de dialogue. Le rôle des chercheurs-accompagnateurs consiste aussi à créer des conditions pour faire éclore la créativité des coenseignants. Sa présence officialise la discussion, il est témoin des échanges et des pistes envisagées.

Dans le cas où les enseignantes ont plus de difficultés à imaginer une autre manière d'aborder certains contenus, le rôle des chercheursaccompagnateurs est ici d'apporter des idées nouvelles, issues de remueméninges, des pratiques issues de la recherche ou encore des exemples d'expériences vues ailleurs, dans d'autres classes de coenseignement. L'objectif est ici d'élargir au maximum le champ des possibles pour permettre ensuite aux coenseignantes d'innover dans leurs pratiques enseignantes. II importe aussi d'accepter, dans ce moment, toutes les idées, même mauvaises, sans porter de jugement. Ensuite, on pourra sélectionner les plus porteuses et réalistes. Par exemple, la note d'observation 2 illustre ce souhait de modifier des pratiques routinières :

Après un certain temps, deux coenseignantes de français trouvaient qu'elles étaient revenues à des pratiques d'enseignement plus traditionnelles. Elles ont demandé à la chercheuse-accompagnatrice de se joindre à elles pour réfléchir aux moyens possibles de créer une tâche en compréhension de lecture impliquant, par la suite, l'écriture d'un texte descriptif. La discussion porte d'abord sur leur intention pédagogique, puis sur les types de textes à utiliser et sur la façon de stimuler les élèves en liant la lecture, l'écriture et l'oral. Plusieurs idées jaillissent à partir de textes choisis: travailler le vocabulaire; faire un atelier sur le surlignement ; faire un schéma en dyade 


\section{$\&$}

\section{REVUE HYBRIDE DE L'ÉDUCATION}

et l'expliquer à une autre dyade; proposer différentes stations où les élèves doivent trouver des mots riches sur un thème précis; faire une courte description à l'aide des mots trouvés; un élève lisant sa description à l'autre, qui doit tenter de la dessiner; etc. II est décidé qu'un atelier en quatre stations sera proposé au prochain cours. Les coenseignantes seront postées dans deux des quatre ateliers afin de réguler les apprentissages des élèves ciblés.

Ainsi, les chercheurs-accompagnateurs peuvent aider les coenseignants dans les différents types de coplanification (didactique, pédagogique, orthopédagogique et structurelle). Toutefois, cet étayage a pour finalité de disparaitre à court ou moyen terme.

À d'autres moments, les chercheurs-accompagnateurs avalisent la mise en œuvre de pratiques innovantes et ce qui permet aux coenseignantes de voir leur collaboration sous un angle nouveau. Par exemple, une jeune enseignante vient de reprendre la tâche d'une enseignante partie à la retraite qui lui a laissé son matériel afin qu'elle puisse assurer la continuité (extrait 3). Elle accueille, comme son prédécesseur, une enseignante-ressource en classe pour faire du coenseignement.

Enseignant titulaire : Je ne me sens pas à l'aise d'enseigner de manière traditionnelle à ces élèves-là. Je le vois: ils s'ennuient, ils se désorganisent.

Enseignant-ressource : Qu'est-ce tu voudrais faire?

Enseignant titulaire : Faire des projets, des activités plus ludiques.

Enseignant-ressource : Qu'est-ce qui t'en empêche?

Enseignant titulaire : Ben, je vois que les autres enseignants ne travaillent pas comme ça. Je me demande si je peux... qu'est-ce qu'ils vont dire les autres?

Accompagnateur : Si on s'amusait à imaginer ce que vous pourriez faire, ce serait quoi?

Enseignant titulaire : Des ateliers, on pourrait utiliser les ordinateurs, ils pourraient créer des bouts de cours et se les enseigner...

Enseignant-ressource : Moi, je travaille beaucoup comme ça dans ma classe, j'ai plein d'idées... 


\section{$\&$}

\section{REVUE HYBRIDE DE L'ÉDUCATION}

L'extrait 3 reflète un questionnement qui revient souvent : Les enseignants d'un même niveau, d'une même discipline, doivent-ils enseigner de la même façon? Cela témoigne du besoin de se faire reconnaître au sein d'une équipe disciplinaire et de prévenir un rejet de la part des autres membres. Les chercheurs-accompagnateurs, dans ce contexte, peuvent encourager l'innovation et proposer un suivi pour réguler l'action.

\subsection{Dialogue cogénératif critique}

Le dialogue cogénératif critique est observable par les chercheursaccompagnateurs, à deux moments : soit juste après un cours qui vient d'être donné, par concertation informelle, soit lors d'une rencontre plus formelle, plus tard dans la journée ou la semaine. Dans le premier cas, c'est souvent à ce moment que les deux enseignantes se rejoignent, après que les élèves aient quitté la classe, pour discuter "à chaud » de l'activité. Ils profitent de la pause d'une quinzaine de minutes entre chaque cours pour ce faire. II s'agit de concertations informelles. La fin d'une activité avec un groupe est ainsi l'occasion d'une rencontre, parfois très courte, entre coenseignants pour donner une rétroaction de l'activité, planifier la suite, donner une information sur un élève (son travail, son attitude, une situation personnelle, etc.). Des exemples d'amorce de questionnements relevés lors de ces microconcertations par les enseignants sont de l'ordre de : " ça s'est bien passé !? », « la prochaine fois, il ne faudrait pas oublier de... » ou encore «Y a super bien (ou mal) travaillé aujourd'hui». Ces microconcertations prenant place quasi systématiquement en cours ou à la fin d'une période de coenseignement, les chercheurs-accompagnateurs ne peuvent y assister que lors de séances d'observations en classe.

La note d'observation 3 , où ce type de concertation informelle après une activité est illustrée, permet de bien saisir cette interaction entre coenseignants.

Suite à une activité nouvelle que viennent d'expérimenter deux coenseignantes, celles-ci se rejoignent quelques minutes près du bureau de l'enseignant titulaire pour discuter de l'activité qu'ils viennent de faire (jeu d'énigmes mathématiques utilisant les contenus du programme). Elles se montrent très satisfaites de cette activité de manière générale. Selon elles, la participation a été bonne et bien meilleure que d'habitude pour ce type de contenu. L'ER cite ensuite au titulaire le nom des élèves qui ont "enfin compris! "Elles confrontent ensuite leurs observations sur ces élèves et ceux qui ont encore des difficultés. Elles envisagent, pour le prochain cours, de faire un coenseignement alternatif dans lequel les quatre élèves en difficulté recevront une remédiation d'une vingtaine de minutes en début 


\section{$\&$}

\section{REVUE HYBRIDE DE L'ÉDUCATION}

de cours, offerte par l'ER. Elles soulignent également certaines pistes d'amélioration (temps plus court, composition des groupes, donner un temps équitable à tous, etc.).

Comme on le voit dans cette note observation, le dialogue critique est la base d'un dialogue cogénératif de remue-méninge dans bien des cas. L'évaluation d'une activité, faite par les coenseignantes, mène à des prises de décisions basées sur l'expérience pour la suite des apprentissages. Ici, le rôle des chercheurs-accompagnateurs est principalement d'observer cette évaluation «à chaud» et de poser, éventuellement, quelques questions uniquement pour donner un sens à certains aspects observés en classe.

Ainsi, il arrive que les chercheurs-accompagnateurs jouent plutôt un rôle de témoin, ses observations peuvent être mises à profit lors d'une rencontre formelle subséquente pour relancer la discussion sur les apports des ajustements apportés. Les réponses fournies par les coenseignants servent à mieux cerner les enjeux organisationnels, pédagogiques, didactiques ou relationnels sous-jacents à la collaboration. On peut prendre également la mesure des ajustements au niveau du dialogue cogénératif de gestion et prévoir d'éventuelles situations demandant un dialogue cogénératif directif.

Pour le second moment du dialogue cogénératif critique, dans le cadre de concertations plus formelles (ex. : lors de réunions organisées entre enseignants), les coenseignants discutent «à froid» de difficultés rencontrées, de solutions ou d'ajustements à apporter. Ces réunions servent, d'une part, suite à la macroplanification réalisée lors du dialogue cogénératif de gestion, d'apporter des ajustements, une régulation de cette macroplanification. D'autre part, les réunions de dialogue cogénératif critique servent à apporter des modifications, notamment à des activités d'enseignement-apprentissage, à la gestion de classe ou aux routines de la classe (ex. : 10 minutes de lecture quotidienne, les devoirs, la synthèse de fin de cours, etc.). L'extrait 4 illustre ce dialogue qui traite des limites perçues quant au contenu ou au regard des pratiques d'enseignement mises en œuvre. L'enseignante titulaire a dû modifier la séquence de ses cours pour que la présence de l'enseignante-ressource soit optimisée. Leur échange traduit les compromis qui ont été faits et les bénéfices perçus chez les élèves.

Enseignant-ressource : Tu as dû changer ta planification avec ma venue.

Enseignant titulaire : Absolument. Il y a eu plus de périodes consacrées à l'écriture, aussi, que dans mes années antérieures; ça fait que j'ai pu prendre du retard dans mes autres... en lecture, en grammaire. Là, je pédale, je dirais. Je veux me rendre à la fin de l'année comme les autres. Donc moi, j'ai peut-être pris plus de périodes pour ça... c'est peut-être 


\section{$\&$}

\section{REVUE HYBRIDE DE L'ÉDUCATION}

bien... faut pas que je prenne ça comme un handicap parce que ç'a été bon pour les élèves. Je pense qu'au bout de la ligne, les élèves sont gagnants.

Accompagnatrice : Quels sont les indicateurs que vous avez perçus pour être capables de dire : ça été bon pour les élèves?

Enseignant titulaire : Ils étaient plus autonomes. Et pour la structure, le plan du texte courant, les différentes parties mieux assimilées. Je l'ai vu dans la pratique. C'était vraiment bien maîtrisé. Les organisateurs textuels étaient là, l'idée principale de chaque aspect était là.

Enseignant-ressource : C'est le fait qu'on a été capable de voir tout le monde. T'sais, dans une période, à deux, on voyait tout le monde.

Dans ce dernier extrait, la prise de recul sur les activités, permise par les chercheurs-accompagnateurs, a généré une réflexion sur l'action et permet d'apprécier ce qui a été réalisé. Bien que leurs interventions soient différentes de celles faites habituellement, les deux coenseignantes identifient des effets positifs. Elles commencent à utiliser des critères pour analyser les effets de leur enseignement. Le rôle des chercheursaccompagnateurs n'est pas d'apporter des points critiques, mais plutôt, grâce aux observations, de les amener à réfléchir à différents aspects qu'elles n'ont pas perçus ou sur lesquels peu d'importance a été mise. Ainsi, les chercheurs-accompagnateurs ne sont pas des évaluateurs, mais plutôt ceux qui peuvent identifier des pistes de réflexion critique.

\subsection{Dialogue cogénératif directif}

Ce dialogue permet aux coenseignants de s'exprimer sur un manque d'égalité et/ou d'équité dans les rôles et de recadrer la relation. II s'agit plus prosaïquement de régler des conflits, problèmes ou insatisfactions entre coenseignants. La note d'observation 4 exemplifie ce type de dialogue cogénératif directif :

En début d'année scolaire, les observations de deux enseignantes (une titulaire en français et une enseignante-ressource qui enseigne une autre matière) montraient une participation assez faible de l'ER dans la planification et la prestation de la situation d'enseignement/apprentissage, alors que cette dernière exprimait le désir d'une plus grande implication. Les entretiens d'accompagnement ont montré que l'enseignante titulaire avait du mal à partager avec l'ER les tâches de planification et d'enseignement, car elle jugeait que sa collègue n'avait pas les compétences didactiques. Elle lui réservait donc essentiellement un rôle de soutien en laissant peu de place à d'autres interventions. Les 


\title{
8
}

\section{REVUE HYBRIDE DE L'ÉDUCATION}

\begin{abstract}
chercheurs-accompagnateurs ont d'abord rappelé les quatre types de planification, puis que l'espace de négociation, s'il ne comprenait pas la planification didactique, incluait toutefois les planifications pédagogiques, orthopédagogiques et structurelles. Cette réflexion a conduit à redéfinir le rôle de l'ER vers, d'une part, une participation plus grande de cette dernière et, d'autre part, une différenciation plus importante des contenus d'enseignement.
\end{abstract}

Le dialogue cogénératif directif prend la forme de rencontres formelles où les enseignants souhaitent aborder ce sujet plus délicat et lors desquelles les chercheurs-accompagnateurs interviennent comme facilitateurs. II soutient les coenseignants, proposent des tours de paroles, s'assurent que les personnes se sentent respectées et proposent, lorsque jugé nécessaire, des pistes de solution pour faire avancer les coenseignants dans leurs réflexions. À la différence du dialogue cogénératif de gestion qui vise à prévoir les problèmes et du dialogue critique qui permet des microajustements, le dialogue directif permet de remédier à des problèmes, conflits ou insatisfactions plus importants ou plus ponctuels entre enseignants.

L'extrait 5, où l'enseignante-ressource a besoin de trouver sa place dans la relation et se sent à l'écart lorsqu'il s'agit de planifier les activités et le contenu, montre le rôle des chercheurs-accompagnateurs qui peut consister à rappeler des fondements théoriques pour rétablir la collaboration et une certaine légitimité dans les rôles assumés.

Accompagnateur : Est-ce qu'à un moment donné tu as énoncé "moi je veux travailler là-dessus, il faut que tu m'aides ? ”

Enseignant-ressource : Bien oui, je l'aidais. Mais moi, avec le recul, et c'est ça que j'ai fini par lui dire... toi tu prépares tout et moi je ne fais rien. T'sais ! J'y vais, je t'accompagne, c'est bien beau, mais...

Accompagnateur : Toi, tu souhaitais t'impliquer.

Enseignant-ressource: C'est l'évolution de l'affaire parce que j'ai commencé avec une autre enseignante; elle a toute la planification dans sa tête. Elle ne m'aurait pas demandé de créer une activité. Tu sais, j'ai commencé à créer avec É. et on a continué comme ça.

Enseignant titulaire : Je pense que tu as eu pitié de moi, un peu aussi parce que j'étais complètement dans le jus! Tu disais "donne-moi du travail, je vais t'aider». 


\section{8}

\section{REVUE HYBRIDE DE L'ÉDUCATION}

Enseignant-ressource : Puis moi des fois, elle faisait des affaires et je lui disais " $t u$ as fait plein de travail, j'aurais pu en faire moi aussi ! J'aurais pu t'aider. Dis-le moi ! »

Enseignant titulaire : Ce n'est pas évident... à un moment donné elle me demandait : "qu'est-ce qu'on fait cette semaine? ?. Je répondais : "On est en écriture. Je peux peut-être t'envoyer ceux qui ont terminé". Mais c'est vrai qu'elle disait "oui, mais là, je ne sers à rien". On n'avait pas encore développé ce désir-là et on n'était pas capable de dire : qu'est-ce que tu pourrais faire en attendant pour nous avancer sur un autre projet.

Concernant l'accompagnement des chercheursaccompagnateurs, ce dernier extrait montre que la demande d'aide à autre personne n'est pas évidente pour des enseignants du secondaire. Le coenseignement les amène, d'une part, à varier leurs stratégies d'enseignement et, d'autre part, à inclure l'autre enseignant dans cette planification. Ainsi, ils ont besoin de suggestions et d'accompagnement pour y parvenir. Cela peut également signifier que le dialogue cogénératif de gestion n'a pas été ouvert et n'a pas permis aux deux enseignants de bien définir leur rôle. Dans ce cadre, les chercheurs-accompagnateurs, comme tiers modérateurs, peuvent permettre l'ouverture de ce dialogue ainsi qu'une redéfinition des rôles et des rapports des forces entre les enseignants.

\subsection{Dialogue cogénératif réflexif}

Le dernier type de dialogue cogénératif s'opère, comme vu plus haut, lorsque les coenseignants utilisent les données recueillies pour prendre des décisions concernant les orientations futures. II permet la régulation à partir de critères définis qui serviront de balises pour apprécier les résultats obtenus et ajuster le tir.

Dans la première situation, celle d'un cours de mathématiques, les deux enseignants (un de mathématiques et un enseignant-ressource dans une autre matière) s'interrogeaient sur la suite à donner à une évaluation formative avant un examen (opérations avec les naturels). Celle-ci montrait qu'environ la moitié de la classe éprouvait encore des difficultés pouvant conduire à un échec des élèves. De plus, les différentes observations réalisées jusqu'alors avaient montré que l'ER était le plus souvent dans une situation d'observateur ou de soutien aux élèves. II s'agissait ainsi également de diversifier les modes de collaboration. L'idée de départ était de faire une révision pour tout le groupe. Les chercheurs-accompagnateurs ont alors amené les coenseignants à s'interroger sur la différenciation 


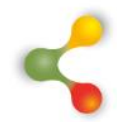

\section{REVUE HYBRIDE DE L'ÉDUCATION}

possible dans ce cadre. Ils ont également donné un exemple de situation à propos des nombres naturels, $v u$ dans une autre classe de coenseignement $d u$ même niveau. La discussion qui a suivi a conduit les coenseignants à se partager le groupe en deux, selon une configuration d'enseignement parallèle: le groupe d'élèves en difficulté recevant un nouvel enseignement de cette matière par l'ER, tandis que les autres travaillaient à deux, en autonomie, dans une tâche d'application avec un habillage ludique inspirée de l'exemple donné par les chercheursaccompagnateurs. L'enseignant titulaire pouvait alors se partager entre les deux groupes (note d'observation 5).

Ce type de dialogue peut s'observer durant les rencontres de planification, en cours d'apprentissage ou en fin d'étape, selon les enseignants. II donne lieu à un dialogue coopératif réflexif au cours duquel les coenseignants discutent d'approches efficaces permettant de s'appuyer sur les connaissances des élèves pour favoriser leur réussite. II s'agit essentiellement de concertations formelles souvent réalisées en présence des chercheurs-accompagnateurs, mais qui pourraient se tenir lors de rencontres collaboratives en équipe-école ou dyade.

Si les données quantitatives (résultats des élèves) sont des indicateurs pertinents pour ajuster les pratiques, les données qualitatives (commentaires, motivation, sentiment de contrôle face à la tâche) servent aussi à apprécier l'engagement des élèves, le climat de la classe ainsi que la relation enseignant-élève. Ce dialogue traduit une prise de recul de la part des coenseignants en vue de prendre des décisions concernant les orientations futures (extrait 6). Ici, les coenseignants se servent de commentaires des élèves pour ajuster leur enseignement.

Enseignant titulaire: C'était vraiment trippant. C'était vraiment très plaisant. Et lire les commentaires, c'était plaisant aussi. Ça fait du bien.

Accompagnateur : Et ça, tu pourrais peut-être en parler un peu. C'est quoi ça, les commentaires? C'est un moyen de régulation pour toi ?

Enseignant titulaire : C'est K. qui m'a amenée ça.

Enseignant-ressource : Moi, je fais souvent ça quand ils ont à travailler en équipe à long terme. Je les fais s'autoévaluer, évaluer leurs pairs, et j'en tiens compte évidemment dans mon évaluation. Donc, moi, c'est quelque chose que je lui ai suggéré. Et en éthique, je le fais souvent: "bon, comment on aurait pu faire ça ? Où est-ce que ça a accroché ? Qu'est-ce que vous pensez qu'on aurait pu faire ? " Et en général, ils répondent. Ils 


\section{$\&$}

\section{REVUE HYBRIDE DE L'ÉDUCATION}

ont vraiment des bonnes idées. Ils répondent à ça, et sincèrement; ce qu'ils ont préféré, ils le disent et ce qu'ils ont moins aimé, ils le disent aussi !

Accompagnateur : Une critique constructive.

Enseignant-ressource : Moi, j'aime ça la critique. Si t'aimes ou t'aimes pas ça, dis-le-moi! C'est de même qu'on s'améliore!

Enseignant titulaire : Donc nous, on s'est servi des commentaires pour créer des nouvelles activités. Pour aller les chercher, les élèves.

Ce dernier extrait montre que les chercheurs-accompagnateurs, dans ce cadre, n'interviennent pas tellement dans les constats qui font suite à ces évaluations, mais plutôt, si le besoin se fait sentir, sur les suites à donner à ces constats. Dans ce sens, ce travail s'approche plus d'un dialogue cogénératif de remue-méninge.

En somme, les coenseignants, dans ces notes d'observation et dans cet extrait, se sont basés sur d'une part, une évaluation sommative diagnostique et d'autre part, sur une évaluation formative pour réguler leur pratique. Le point de vue des élèves est considéré et fait l'objet d'une concertation entre les enseignants.

\section{Discussion}

Le coenseignement est un dispositif reconnu par plusieurs chercheurs comme une réponse positive à l'inclusion scolaire (Friend et Cook, 2013 ; Murwaski, 2009). Cependant, il exige des enseignants de haut niveau de collaboration qui s'exprime dans trois temps pédagogiques interreliés soit la coplanification, le coenseignement et la coévaluation (Murwaski, 2009). C'est à travers le dialogue que le préfixe "co » prend son sens. Or, paradoxalement, les enseignants du secondaire sont habitués à un fonctionnement « en silo » (Maroy, 2006). Cela est attribuable à divers facteurs dont une culture scolaire où les enseignants sont des spécialistes disciplinaires et ont une faible connaissance des pratiques pédagogiques mutuelles, une organisation scolaire qui laisse peu de temps pour la concertation, une tâche qui combine des périodes d'enseignement individuel et de coenseignement et ce, dans différentes disciplines et différents niveaux (Tremblay et Granger, accepté). II apparait donc nécessaire que les coenseignants prennent le temps de s'apprivoiser et ait la possibilité d'échanger avant, pendant et après chaque période de coenseignement. Les recherches répertoriées rapportent que le dialogue cogénératif est un outil de discussion permettant de faire évoluer les acteurs impliqués et de favoriser des changements (Beltramo, 2017; Scantlebury, 2008 ; Stith et Roth, 2010).

Les types de dialogues illustrés dans cet article apportent un éclairage sur le contexte d'utilisation. À titre d'exemple, le dialogue 


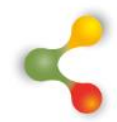

\section{REVUE HYBRIDE DE L'ÉDUCATION}

cogénératif de gestion s'exprime notamment en contexte de collaboration directe soit pendant l'enseignement. Les observations permettent de relever des microconcertations et des signes non verbaux utilisés pour gérer et ajuster l'activité en cours. À l'inverse, les dialogues de remueméninge, critique, directif et réflexif sont davantage observés lors de rencontres formelles, car ils impliquent que les coenseignants échangent, se questionnent, génèrent de nouvelles idées, résolvent des situations problèmes et prennent du recul sur leur pratique (Tobin, 2006). En ce sens, le dialogue cogénératif serait un levier pour le développement professionnel des coenseignants (Beltramo, 2017). II peut faire prendre conscience des rôles joués par chacun et des effets sur l'apprentissage des élèves (Tobin et Roth, 2006). II porte la discussion sur les besoins des élèves et encourage les coenseignants à discuter des moyens différenciés pour organiser la classe, créer des situations d'enseignementapprentissage innovantes reliées aux contenus du programme (Scantlebury et al., 2008).

Les exemples présentés montrent que le rôle des chercheursaccompagnateurs varie donc selon le type de dialogue. Dans certains cas, leur rôle se limite à prendre note et à orienter la discussion, au besoin, vers certaines thématiques en laissant les coenseignants trouver seuls les réponses. Dans d'autres cas, il a un rôle plus actif en aidant les coenseignants à trouver des solutions aux problèmes qu'ils rencontrent. Les chercheurs-accompagnateurs peuvent s'appuyer sur cette typologie pour orienter leur intervention directe ou indirecte (ou leur non-intervention) sur l'ensemble des types de dialogue. II peut ainsi identifier le type de contenus abordés, le circonscrire, informer les coenseignants sur les enjeux, etc. Le dialogue cogénératif constitue un cadre à l'accompagnement. De plus, les résultats montrent que ce dialogue permet d'inclure les chercheurs-accompagnateurs comme participant actif. Les chercheurs-accompagnateurs ne doivent pas toujours animer ou parler, mais il doit constamment être présent. Un accompagnement planifié pourrait tenir compte de ces types de dialogues et les générer selon le contexte. Ces types de dialogues peuvent aussi servir à alimenter des dispositifs de recherche-action tels que le groupe de discussion ou pour soutenir un entretien de confrontation croisé, dans le cas où les coenseignants acceptent de se filmer.

Comme vu plus haut, pour conduire à une meilleure compréhension des besoins des élèves, le dialogue cogénératif doit s'inscrire dans une relation dialectique impliquant trois aspects (Stith et Roth, 2006, 2008) : l'activité de coenseignement en elle-même, le corespect et la coresponsabilité des enseignants (Scantlebury et al., 2008). Le rôle des chercheurs-accompagnateurs est ainsi guidé par ces trois aspects du dialogue cogénératif. En effet, discuter ensemble du coenseignement permet de générer une vision commune des activités d'enseignement-apprentissage, mais également de la gestion de classe pour répondre aux besoins des élèves. À titre d'exemple, les extraits 


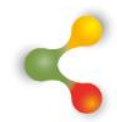

\section{REVUE HYBRIDE DE L'ÉDUCATION}

présentés montrent que le choix des configurations du coenseignement traduit les préoccupations mutuelles des deux enseignants et renseignent sur leur niveau d'interdépendance. Certains coenseignants peuvent se sentir menacés et vivre cette proximité avec l'autre comme une intrusion dans leur espace professionnel. À cet égard, le dialogue cogénératif basé sur l'activité de coenseignement ouvre sur des pistes d'accompagnement ou de questionnement pour les chercheurs-accompagnateurs qui visent le développement d'une compétence collective à collaborer : faire ensemble pour mieux agir. Le développement d'une pratique réflexive commune s'amorce grâce à un objet conjoint sur lequel poser les bases de l'interaction.

Le deuxième aspect du dialogue cogénératif touche le corespect qui témoigne du respect mutuel entre les coenseignants qui favorise la communication et crée un environnement ouvert à la critique constructive, au partage et à la génération de nouvelles idées, et potentiellement une expérience de coenseignement productive. Ce corespect représente la base du coenseignement, une condition essentielle dans la relation entre enseignants et entre enseignants et chercheurs-accompagnateurs. Dans le cas qui nous occupe, les coenseignants sont volontaires. L'intensivité de ce coenseignement, c'est-à-dire, $50 \%$ à $100 \%$ du temps de classe dans un groupe donné, permet, par ailleurs, de faciliter la compatibilité entre enseignants ainsi que le développement d'une relation intime au niveau professionnel et personnel. Ce corespect apparait ainsi tant comme une condition que comme un résultat du coenseignement. Pour les chercheursaccompagnateurs, il peut s'agir de relever des faits observés qui aident les coenseignants à voir qu'ils ont amorcé la discussion et se dire ce qu'ils ressentent, comment ils perçoivent leur action commune dans le but de travailler ensemble. Que ce soit par un échange de regard, d'une simple phrase dédiée à l'autre avant, pendant ou après l'activité, les chercheursaccompagnateurs peuvent questionner l'intention, permettre l'explicitation en vue de mieux accepter les idées de l'autre, lui faire une place dans la classe, dans sa routine et faire exprimer des attentes non formulées. Ce type de routine reprise au fil du temps permet de construire la relation et la collaboration.

En dernier lieu, le dialogue cogénératif permet de traiter de la responsabilité partagée entre les coenseignants. Celle-ci peut se rapporter à divers enjeux relatifs à la classe tels que l'enseignement, les élèves ou encore les résultats de l'enseignement et de l'apprentissage. La coresponsabilité s'exprime davantage dans les dialogues de gestion, critique et directif. Elle englobe une autorité également partagée décrite par Scantlebury et al. (2008) comme un équilibre entre «donner et prendre » par les coenseignants. Cet équilibre n'est pas évident à établir pour tous les partenaires. Dans les notes d'observations et les dialogues cela s'exprime au sujet de la planification des activités (temps accordé pour chaque étape de l'enseignement, la considération des caractéristiques des élèves), la mise en œuvre (selon des commentaires d'élèves, les forces 


\section{$\&$}

\section{REVUE HYBRIDE DE L'ÉDUCATION}

spécifiques de chacun des coenseignants) ou la régulation du coenseignement telle que les observations faites en cours d'activité, la réussite des tâches planifiées, ou encore pour rétablir les rôles de chacun. Pour les chercheurs-accompagnateurs, il s'agit d'encourager la mobilisation et le pouvoir d'action des partenaires dans le but qu'ils développent leur leadeurship comme enseignants.

Parmi les limites relevées, par les enseignants et les chercheursaccompagnateurs, dans le déploiement du dialogue cogénératif, le peu de temps consacré aux échanges dans l'horaire rend complexe la tenue de ce type de dialogue. De plus, les coenseignants gardent peu de traces des contenus abordés, ce qui a un effet sur le suivi consacré aux échanges et à leur prise en compte effective dans la classe. En effet, les temps de concertations, les objets et les formes ne sont pas les mêmes. Ces données ne sont pas toujours accessibles aux chercheursaccompagnateurs, surtout in situ (ex. : les concertations informelles). De plus, il faut prendre en considération que leur présence, même passive, a un effet sur les coenseignants. Les chercheurs-accompagnateurs influencent ce qu'ils observent. Dans le cadre du dialogue cogénératif impliquant les chercheurs-accompagnateurs en recherche-action, le but est clairement d'avoir un effet sur la pratique pédagogique, tout en prenant soin de laisser la liberté aux enseignants de faire leurs choix, de définir leurs rôles et de penser l'innovation à leur manière. Ainsi, le rôle des chercheurs-accompagnateurs est non pas normatif (ce qui est un bon coenseignement et ce qui n'en est pas un), mais celui d'un catalyseur qui permet à chaque binôme de réaliser son potentiel. II ne s'agit ainsi pas de donner le cadre d'une collaboration idéale, mais plutôt celui d'un idéal de collaboration. Puisqu'au secondaire le coenseignement intensif à temps plein est peu habituel, des communautés de pratiques ou d'apprentissage professionnels peuvent servir de dispositif structurant pour rassembler les enseignants et les enseignants-ressources qui coenseignent. Le dialogue cogénératif peut alors servir de base à l'établissement d'une vision commune qui guidera la planification, l'action et la régulation de l'action (Cook et Tankersley, 2013). 


\section{REVUE HYBRIDE DE L'ÉDUCATION}

\section{Références}

Beers, J. (2005). The role of coteaching in the development of the practices of an urban science teacher. Dans W. Roth et K. Tobin (dir.), Teaching together, learning together (p. 79-96). New York : Peter Lang.

Bélanger, S. (2010). Attitudes des différents acteurs scolaires à l'égard de l'inclusion. Dans N. Rousseau (dir.), La pédagogie de l'inclusion scolaire (2e éd., p. 111-132). Presses de l'Université du Québec.

Beltramo, J. (2017). Developing adaptive teaching practices through participation in cogenerative dialogues. Teaching and Teacher Education, 63, 326-337.

Bergeron, G. et Granger, N. (2016). Retombées de deux projets de recherche-action impliquant une pratique inclusive au secondaire. Education \& Sociology, 3(6), 161-177.

Boudreault, P. et Kalubi, J.-C. (2006). Animation de groupes: Une démarche réflexive d'analyse. Éditions Carte Blanche.

Cook, B.G. et Tankersley, M.G (2013). Research-based practices in special education. Pearson Education.

Emdin, C. (2007). Exploring the contexts of urban science classrooms: Part 1 : Investigating corporate and communal practice. Cultural Studies of Science Education, 2, 319-350.

Engeström, Y. (1987). Learning by expanding: An activity-theoretical approach to developmental research. Orienta-Konsultit.

Engeström, Y. (1994). Teachers as collaborative thinkers: Activitytheoretical study of an innovative teacher team. Dans I. Carlgren, G. Handal et S. Vaage, (dir.), Teachers' Minds and Actions: Research on Teachers' Thinking and Practice (p. 43-61). The Falmer Press.

Engeström, Y. (2001). Expansive learning at work: toward an activity theoretical reconceptualization. Journal of Education and Work, 14(1), 133-156.

Fédération des syndicats de l'enseignement [FSE].(2015). Fédération des syndicats de l'enseignement: Convention collective. http://lafse.org/vos-droits/conventions-collectives/conventioncollective-nationale/

Fennick, E. et Liddy, D. (2001). Responsibilities and preparation for 


\section{REVUE HYBRIDE DE L'ÉDUCATION}

collaborative teaching: co-teacher's perspectives. Teacher Education and Special Education, 24, 229-240.

Friend, M. P. et Cook, L. (2007). Interactions : Collaboration skills for school professionals. Longman.

Friend, M. et Cook, L. (2013). Interactions : Collaboration skills for school professionals ( $5^{\mathrm{e}}$ éd.), Pearson Education.

Gallo-Fox, J. et Scantlebury, K. (2006, avril). Coteaching: A professional development model for cooperating teachers. Paper presented at American Educational Research Association Annual Meeting, San Francisco, CA.

Granger, N. et Dubé, F. (2015). Définir la fonction d'enseignant-ressource dans le secondaire : une recherche-action formation. La nouvelle revue de l'adaptation et de la scolarisation, 2(70-71), 121-136.

Granger, N. et Tremblay, P. (2019). Satisfaction des enseignantsressources à l'égard des rôles et des fonctions pour soutenir la réussite des élèves à besoins spécifiques. McGill Journal of Education, 54, 132-150.

Hang, Q. et Rabren, K. (2009). An examination of co-teaching: Perspectives and efficacy indicators. Remedial and Special Education, 30(5), 259-268.

Hartnett, J., Weed, R., McCoy, A., Theiss, D. et Nickens, N. (2013). Coteaching: A new partnership during student teaching. SRATE Journal, 23(1), 5-12.

Hugon, M.A. et Seibel, C. (1988). Recherches impliquées, recherches action : Le cas de l'éducation. De Bœck Université.

Kalubi, J.-C. (2015). Portrait de la situation ds EHDAA au Québec (20002013): une analyse multidimensionnelle des caractéristiques, besoins, réseaux de soutien et piste d'innovation. Université de Sherbrooke et Fondation Lucie et André Chagnon.

Kampwirth, T. J. (1999). Collaborative consultation in the schools: Effective practices for students with learning and behavior problems. Merrill.

LaVan, S. K. et Beers, J. (2005). The role of cogenerative dialogue in learning to teach and transforming learning environments. Dans K. Tobin, R. Elmesky et G. Seiler (dir), Improving urban science education: New roles for teachers, students and researchers ( $p$. 147-164). Rowman and Littlefield. 


\section{REVUE HYBRIDE DE L'ÉDUCATION}

Leont'ev, A. N. (1978). Activity, consciousness and personality. Prentice Hall.

Maroy, C. (2006). École, régulation et marché : une comparaison de six espaces scolaires locaux en Europe. Presses universitaires de France.

Ministère de l'Éducation, de l'Enseignement Supérieur et de la Recherche [MEESR]. (2015). Statistiques de l'éducation: Éducation préscolaire, enseignement primaire et secondaire (Édition 2014). Gouvernement du Québec.

Ministère de l'Éducation, du Loisir et du Sport [MELS]. (2014). Statistiques de l'éducation: enseignement primaire, secondaire, collégial et universitaire. Gouvernement du Québec.

Ministère de l'Éducation du Québec [MEQ]. (1999). Politique de l'adaptation scolaire: Une école adaptée à tous ses élèves. Gouvernement du Québec.

Murata, R. (2002). What does team teaching mean? A case study of interdisciplinary teaming. The Journal of Educational Research, 96(2), 67-77.

Murawski, W. M. (2009). Collaborative teaching in secondary schools: Making the co-teaching marriage work! Corwin Press.

Murawski, W. W. et Hughes, C. E. (2009). Response to intervention, collaboration and co-teaching: a logical combination for successful systemic change. Preventing School Failure: Alternative Education for Children and Youth, 53(4), 267-277.

Ploessl, D. M., Rock, M. L., Schoenfeld, N. et Blanks, B. (2010). On the same page: Practical techniques to enhance co-teaching interactions. Intervention in School and Clinic, 45, 158-168.

Prud'homme, L., Dolbec, A. et Guay, M.-H. (2011). Le sens construit autour de la différenciation. Éducation et francophonie, 39(2), 165-188.

Scantlebury, K., Gallo-Fox, J. et Wassel, B. (2008). Coteaching as a model for preservice secondary science teacher education. Teaching and teacher education, 24, 967-981.

Scruggs, T. E., Mastropieri, M. A. et McDuffie, K. A. (2007). Co-teaching in inclusive classrooms: A metasynthesis of qualitative research. Exceptional Children, 73(4), 392-416.

Sileo, J. M. (2011). Co-teaching : Getting to know your partner. Teaching 


\section{REVUE HYBRIDE DE L'ÉDUCATION}

Exceptional Children, 43(5), 32-38.

Siry, C. et Lang, D. (2010). Creating participatory discourse for teaching and research in early childhood science. Journal of Science Teacher Education, 21(2), 149-160.

Smagorinsky, P. (2013). The development of social and practical concepts in learning to teach: A synthesis and extension of Vygtosky's conception. Learning, Culture, and Social Interaction, 2, 238-248.

Stith, I. et Roth, W. M. (2006). Who gets to ask the questions: The ethics in/ofcogenerative dialogue praxis. Forum: Qualitative Social Research, 7(2), 38-57.

Stith, I. et Roth, W. M. (2008). Students in action: Cogenerative dialogues from secondary to elementary schools. Sense Publishers.

Stith, I. et Roth, W. M. (2010). Teaching as mediation: The cogenerative dialogue and ethical understandings. Teaching and Teacher Education, 26, 363-370.

Tobin, K. (2006). Learning to teach through coteaching and cogenerative dialogue. Teaching Education, 17(2), 133-142.

Tobin, K., et Roth, W. (2006). Teaching to learn: A view from the field. Sense Publishers.

Tremblay, P. (2010). Évaluation comparée de la qualité de deux dispositifs d'intervention auprès d'élèves ayant des troubles d'apprentissage [Thèse de doctorat inédite]. Université Libre de Bruxelles, Belgique.

Tremblay, P. et Belley, S. (2017). Cheminement scolaire d'élèves en difficulté adaptation ou d'apprentissage en enseignement secondaire. Revue canadienne de l'éducation, 40(2), 1-30.

Tremblay, P. et Granger, N. (2018). Les enseignants spécialisés au Canada : une analyse comparée. Formation et profession, 26(2), 80-98.

Tremblay, P. et Granger, N. (accepté). Portrait de la fonction d'enseignantressource au Québec: quels services pour quels élèves. Formation et profession.

Trent, S. C., Driver, B. L., Wood, M. H., Parrott, P. S., Martin, T. F. et Smith, W. G. (2003). Teaching and Teacher Education, 19(2), 203-219. 